\title{
Stability Study of Single-abrasive Coupled Flutter in Ultrasonic Vibration Honing
}

\author{
Xiao Qiang Zhang ${ }^{1, a}$, Xi Jing Zhu ${ }^{1, b}$, Ling Long Cheng ${ }^{2, c}$ \\ ${ }^{1}$ School of Mechanical and Power Engineering, North University of China, Taiyuan 03051,China \\ ${ }^{2}$ Shanxi Jincheng Anthracite Mining(Group)King Ding Mining and Machinery CO., LTD., Jincheng \\ 048006, China \\ anuc0741@126.com, bxj3618@262.net, cclljy2011@163.com
}

Keywords: ultrasonic vibration honing, coupled flutter, stability

\begin{abstract}
Flutter, a seriously dynamic and severe self-excited vibration, easily exists in mechanical processing system, is a major factor, impact the workpiece surface quality and machining efficiency. It has been the main research to explore the resource, analysis the mechanism and pursue restraining method at present. On basic of single-abrasive coupled flutter mechanism in ultrasonic vibration honing, established the physical and mathematical coupled flutter model for nonlinear system respectively, then analyzed the relations between the stability and honing parameters, and obtained the best honing condition finally. This paper provide a theoretical basis for to further seeking the strategy for repressing and eliminating the flutter.
\end{abstract}

\section{Introduction}

The ultrasonic vibration honing, a newly processing technology, gains widely employed and develop due to the advantages of lowly temperature, pulse nature, small cutting force and etc. Flutter, a phenomenon accompany with processing, shows two different sides, and affect a serious bad impact, even worse. Whatever, it become the main research mission to analysis the mechanism, seek the effective ways to restrain. This paper is focus on analyzing the stability of flutter, further studying the influence of honing parameter for machining.

The ultrasonic vibration honing, a mechanical processing, use the banding abrasive tools to machine the workpiece, the oilstone is forced vibration and work on the workpiece surface. Three vibration forms are developed, based on the direction of ultrasonic wave, radial vibration, longitudinal vibration and torsional vibration respectively ${ }^{[1]}$, as shown in figure 1 .

\section{Coupled Flutter Model}

Coupled flutter, a kind of flutter in vibration system, caused by two nearly equal stiffness in two different orientations ${ }^{[2]}$. Due to the negative effect, many researchers have devoted themselves to the study, that exploring its develop mechanism and asking for the solving method ${ }^{[3-5]}$.

The sharpe of oilstone is mainly bar, so now presented the coupled flutter model of oilstone and workpiece. Figure 2(a) show the physical model containing spring and damp two free degrees, imposing 


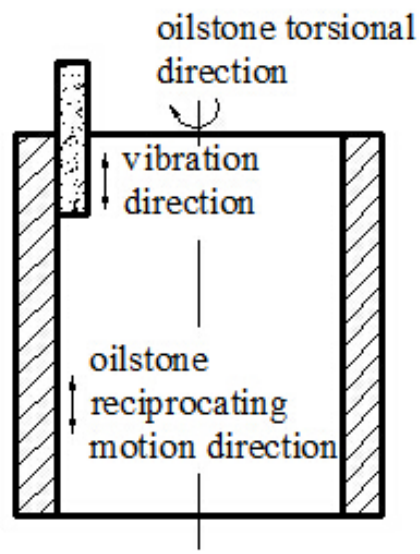

(a)longitudinal vibration

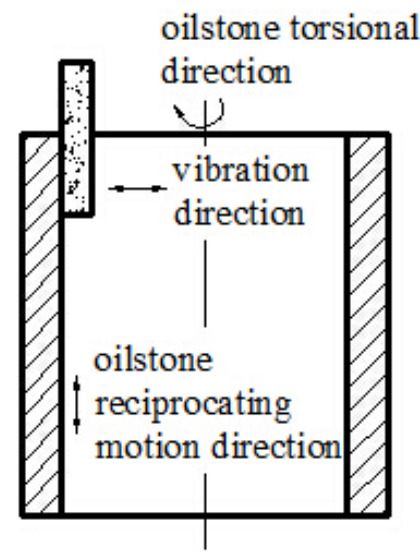

(b)radial vibration

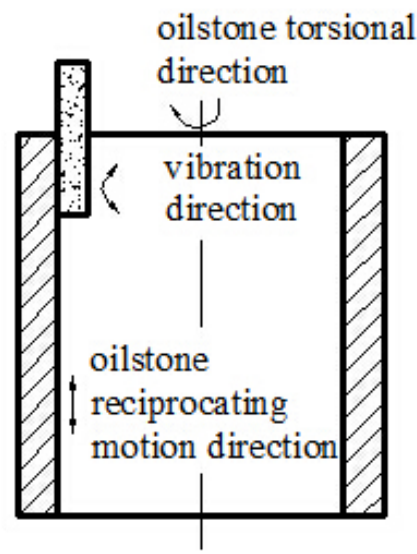

(c)torsional vibration

Fig.1 Processing modes of ultrasonic vibration

On the oilstone. For the system, the differential equation of chatter can be expressed by (1).

$$
\left\{\begin{array}{l}
m \ddot{x_{1}}+c_{1} \dot{x}_{1}+k_{1} x_{1}=-d_{p x_{1}} \\
m \ddot{x_{2}}+c_{2} \dot{x_{2}}+k_{2} x_{2}=-d_{p x_{2}}
\end{array}\right.
$$

Where, $m$ is the equivalent mass of abrasive, $k_{1}$ and $k_{2}$ is the coefficient of elasticity of two orientations respectively, $c_{1}$ and $c_{2}$ is the damping coefficient.

In ultrasonic vibration honing, a single abrasive is equal with a cutting lip. To simplify and establish the simply physical and mathematical model, a serous assumptions for abrasive is given: (1)the abrasive is ruleless on the oilstone; (2)a cone angle is existed; (3)the sharpe of abrasive is asymmetrical spreaded; (4)every abrasive is absolutely homology.

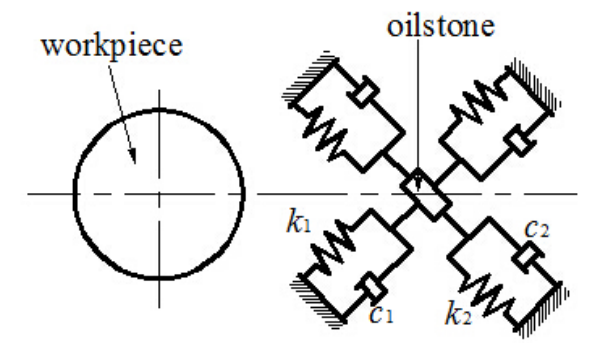

(a)

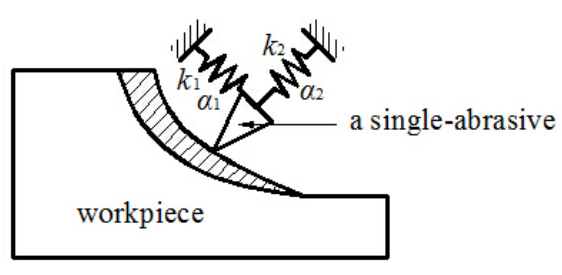

(b)

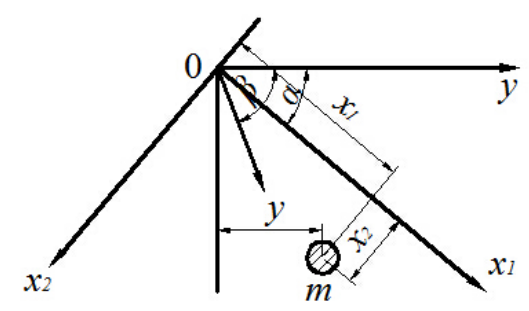

(c)

Fig.2 (a)Two free degrees coupled flutter model

(b) Simply coupled flutter physical model of a single abrasive and workpiece

(c) The mathematical model of abrasive and workpiece coupled flutter

For simply analyzing the model, the damp is ignored in the paper, Figure 2(b) shows the simply physical model. Combined the cutting relationship of abrasive and workpiece, with the generating mechanism of self-excited vibration, converted the physical model to the mathematical model by a way of math, Figure 2(c) shows the mathematical model.

Based on above model, the mathematical differential equation of abrasive and workpiece coupled flutter is expressed as 


$$
\left\{\begin{array}{l}
m \ddot{x_{1}}+\left[k_{1}+k_{s} \cos \alpha \cos (\beta-\alpha)\right] x_{1}-k_{s} \sin \alpha \cos (\beta-\alpha) x_{2}=0 \\
\ddot{x_{2}}+\left[k_{2}-k_{s} \sin \alpha \sin (\beta-\alpha)\right] x_{2}+k_{s} \cos \alpha \sin (\beta-\alpha) x_{1}=0
\end{array}\right.
$$

Where, $k_{s}$ is the pressure variation coefficient of interface in $y$ direction, $\alpha$ is the intersection angle between rigidity spindle $x_{1}$ and y axis, $\beta$ is the intersection angle for cutting force $F$ and $y$ axis.

\section{Stability Analysis}

The especially solution for (2) can be assumed as

$$
\left\{\begin{array}{l}
x_{1}=A_{1} e^{s t} \\
x_{2}=A_{2} e^{s t}
\end{array}\right.
$$

Where, $A_{1}$ and $A_{2}$ are complex conjugate, which mean $x_{1}$ and $x_{2}$ is out of step, $s$ is plural.

Then (2) can be simplified as

$$
\left\{\begin{array}{l}
{\left[m s^{2}+k_{1}+k_{s} \cos \alpha \cos (\beta-\alpha)\right] A_{1}-k_{s} \sin \alpha \cos (\beta-\alpha) A_{2}=0} \\
k_{s} \cos \alpha \sin (\beta-\alpha) A_{1}+\left[m s^{2}+k_{2}-k_{s} \sin \alpha \sin (\beta-\alpha)\right] A_{2}=0
\end{array}\right.
$$

Equation (4) has non-zero solution, so the relation of coefficient can be expressed as

$$
m^{2} s^{4}+m s^{2}\left(k_{1}+k_{2}-k_{s} u_{1}+k_{s} u_{2}\right)+k_{1} k_{2}-k_{1} k_{2} u_{1}+k_{2} k_{s} u_{2}=0
$$

Where, $u_{1}, u_{2}$ is the direction coefficient.

$$
\begin{array}{r}
u_{1}=\sin \alpha \sin (\beta-\alpha)=\frac{1}{2}[\cos (2 \alpha-\beta)-\cos \beta] \\
u_{2}=\cos \alpha \cos (\beta-\alpha)=\frac{1}{2}[\cos (2 \alpha-\beta)+\cos \beta]
\end{array}
$$

For simplified (5), the definition is given.

$$
z=\frac{s}{\sqrt{k_{s} / m}} ; H_{1}=\frac{k_{1}}{k_{s}} ; H_{2}=\frac{k_{2}}{k_{s}}
$$

Then (5) can be defined as

$$
z^{4}+z^{2}\left(H_{1}+H_{2}-u_{1}+u_{2}\right)+H_{1} H_{2}-H_{1} u_{1}+H_{2} u_{2}=0
$$

Further, the express can be given.

$$
A=\frac{1}{2}\left(H_{1}+H_{2}-u_{1}+u_{2}\right) ; \quad B=H_{1} H_{2}-H_{1} u_{1}+H_{2} u_{2} ; \quad E=A^{2}-B
$$

Based on the express, equation (6) can obtained as

$$
z^{4}+2 A z^{2}+B=0
$$

The characteristic solution of (7) can be obtained as

$$
z^{2}=-A \pm \sqrt{E}
$$

Combined above express, equation (8) can be expressed as

$$
s_{1,2}^{2}=\frac{k_{s}}{m}(-A \pm \sqrt{E})
$$

Discussion:

(1)A2 $>B$, which is equal to $E>0$.

( $\mathrm{i}$ ) $\mathrm{A} 2<\mathrm{E}$, it is mean that the system has no vibration motion, and stays in stability.

(ii)A2 $>E$, the system will produce free vibration, but the system can be maintain stability because the damp existed.

(2)A $2<\mathrm{B}$, the system will be continuously be instability and can not be affected by parameters.

(3)A2=B, it could be stay in a critical condition.

Based on analysis, it can be noted that the detailed discussion should be carried out for verifying the state of flutter system. The relation between two direction can be pictured as Figure4 which can be obtained by discussing the connection A and B. It is shown that the system stay stability state in 
machining process from (a) for $A>B$. Moreover, the system is instability from (b) for $A<B$.

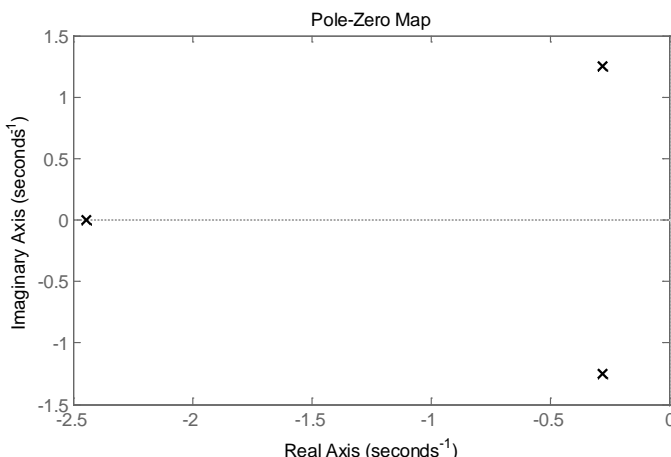

(a) $A^{2}>B$

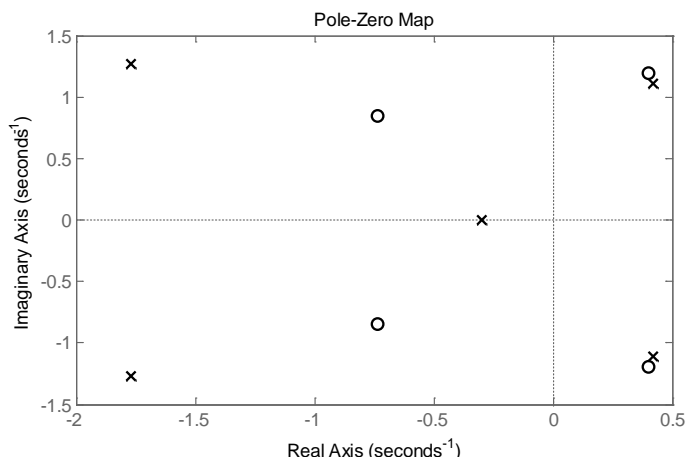

(b) $A^{2}<B$

Figure 4 Stability of flutter system

\section{Acknowledgements}

The authors would like to acknowledgement the financial support supported by National Natural Science Foundation of China(No.50975264), Science and Technology Research Foundation of Shanxi Province(No.2013011024-5).

\section{References}

[1] X J Zhu,Y X Gao,H J Xu. A new ultrasonic vibration machine for honing[J]. International Journal of Computer Application in Technology. 2007,29(2-4):216.

[2] Lu Hongwei,Tang Yanbin,Wu Ya,et al. The Chaotic Nature of Machine Tool Chatter[J]. Huazhong Univ. of SCi.\&Tech. 1995,23(6):105-108.

[3] Wang J Q,Zhu X J,Cheng Q. Study on friction chatter mechanism of power ultrasonic vibration honing based on Stribeck effect[J]. Applied Mechanics and Materials. 2013,328:531-535.

[4] Sun Hai,Yang Lin,Li Hongxin. Sensitive Flutter Parameters Analysis with Respect to Flutter-free Design of Compressor Blade[J]. Procedia Engineering. 2015,99:39-45.

[5] Bojan Gjerek,Radovan Drazumeric,Franc Kosel. Flutter behavior of a flexible airfoil: Multiparameter experimental study[J]. Aerospace Science and Technology. 2014,36:75-86. 